\title{
Powered wind tunnel tests setup of the IRON innovative turboprop aircraft
}

\author{
Danilo Ciliberti ${ }^{1, *}$, Vincenzo Cusati $^{1}$, Pierluigi Della Vecchia ${ }^{1}$, Salvatore Corcione ${ }^{1}$, and \\ Fabrizio Nicolosi ${ }^{1}$ \\ ${ }^{1}$ University of Naples “Federico II", Dept. of Industrial Engineering, Via Claudio 21, 80125, Napoli, \\ Italy
}

\begin{abstract}
This paper describes the powered wind tunnel tests setup of the innovative configuration of the IRON regional turboprop aircraft. The objective of the tests is the evaluation of propulsive effects on aircraft stability and control characteristics. During the setup process, several aerodynamic issues have been anticipated and here illustrated. A scaled engine deck has been derived from the full-scale data provided by the IRON powerplant consortium partner. From two representative flight conditions, the characteristics of the scaled motor as RPM, torque and power have been calculated, providing a choice for the electric motors to install in the test section. The motors' operating voltage and current determined the sizing of the power, acquisition and control system. Similarly, the desired propeller coefficients were the target of a propeller design process, which was performed with XROTOR, MATLAB ${ }^{\circledR}$, XFOIL and validated with RANS analyses. Finally, to directly evaluate the propeller thrust and normal force, motors' supporting structures with load cells have been conceptually designed.
\end{abstract}

\section{Introduction}

This research work is framed in the Innovative turbopROp configuratioN (IRON) project that complies with the European Union topic JTI-CS2-2015-CPW02-REG-01-03 (Green and cost-efficient Conceptual Aircraft Design including Innovative Turbo-Propeller Powerplant), as part of the Clean Sky 2 program for Horizon 2020.

The actual innovative turboprop configuration, which has been shown in several exhibitions partnered with Clean Sky 2, is a three-lifting surfaces aircraft with rearmounted, propeller-driven engines. A summary of the project status, including the last configuration that will be investigated in the wind tunnel, are presented in the companion conference paper [1]. More details are presented in Refs. [2]-[6]. For the sake of brevity, it is here resumed that the innovative configuration attempts to be a competitor of the regional jet, with a load capacity of 130 passengers, a design range of $1600 \mathrm{~nm}$, a cruise Mach number of about 0.65 and a large turboprop engine with a thrust specific fuel consumption $25 \%$ less than a modern high-bypass ratio geared turbofan. The aircraft three-view is shown in Fig. 1.

\footnotetext{
*Corresponding author: danilo.ciliberti@unina.it
} 

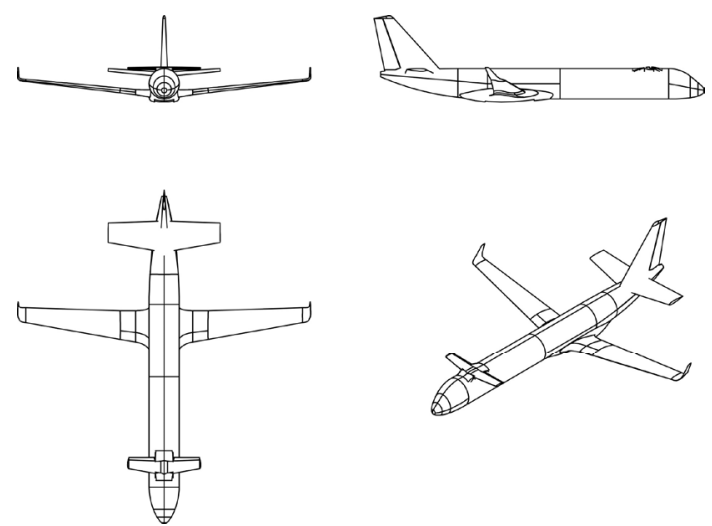

Fig. 1. Three-view and iso-metric view of the IRON innovative aircraft configuration.

In order to validate the stability and control characteristics, wind tunnel tests in prop-off and prop-on conditions have been planned by the end of 2019. To achieve this objective, both the available aircraft model and wind tunnel facility must be upgraded. The aircraft model will be provided with the last versions of the lifting surfaces, including movables. The sizing of the scaled propulsive system as well as the necessary wind tunnel hardware upgrades are presented in this paper.

Section 2 describes the propulsive system scaling tailored to the evaluation of the aircraft stability and control characteristics. Section 3 illustrates the selected electric motor characteristics. Section 4 presents the propeller design process. Section 5 deals with the wind tunnel upgrades for the propulsive tests.

\section{Scaling the propulsive system}

The scale of the aircraft model $(1: 25)$ and the wind tunnel operating speed determine the scale of the propulsive system. Figure 2 shows a rendering of the model in the test section. The engine deck provided by GE Avio has been scaled by applying the thrust similarity, a principle stating that both the model and full-scale propeller have the same coefficients

$$
\begin{aligned}
& J=V_{\propto} /(n D) \\
& C_{T}=T /\left(\rho n^{2} D^{4}\right) \\
& C_{P}=P /\left(\rho n^{3} D^{5}\right)
\end{aligned}
$$

representing the propeller advance ratio (1), thrust coefficient (2) and power coefficient (3).

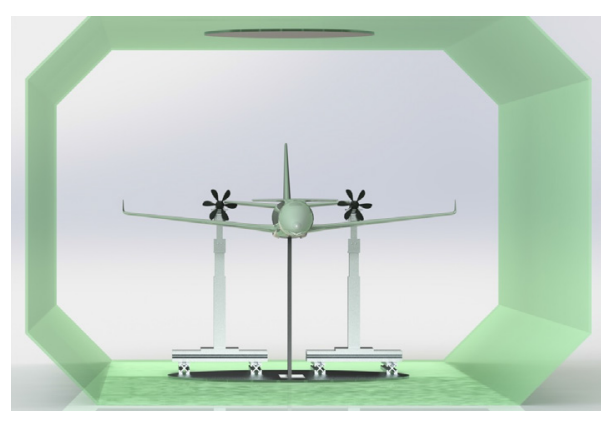

Fig. 2. Rendering of the wind tunnel model $(1: 25)$ in the test section. 
The complete thrust similarity is achieved by matching both $C_{T}$ and $C_{P}$. This is quite difficult to fulfil, since even with an identical, scaled copy of the real propeller, which geometry at time of writing is unknown, the smaller Reynolds number attainable in the wind tunnel limits the maximum values of such coefficients [7]-[10]. Moreover, in a wind tunnel test the flow speed $V_{\propto}$ is usually kept constant, limited only by the maximum power available to the fan or the maximum load tolerated by the strain gage balance. Consequently, the desired advance ratio $J$ is obtained by varying the propeller RPM. For these reasons, the axial and rotational flow speeds are scaled differently: at the same thrust coefficient $C_{T}$ the swirl of the scaled propeller will be different from that generated by the full-scale propeller, hence the power coefficient $C_{P}$ will be different.

As the objective of the wind tunnel test campaign is the evaluation of propulsive effects on aircraft stability and control characteristics, the authors decided to attempt to match the thrust coefficient $C_{T}$ for a few representative flight conditions.

\subsection{Expected propulsive effects on the IRON innovative aircraft}

The theory of aircraft stability and control [11] states that propulsive effects can be divided into direct and indirect effects (Fig. 3). Direct effects are due to the aerodynamic forces exerted by and on the propeller: the thrust $T$ and the normal force $N$. The last is orthogonal to the thrust line and it is generated in non-axial flows. Both produce a pitching moment which value depends on the distance of the aircraft centre of gravity to the thrust line and propeller disk. Indirect effects are related to the propeller slipstream impacting on the tailplane, altering the downwash and sidewash angles, and on the variation of dynamic pressure at the tailplane due to the pressure jump through the propeller disk.

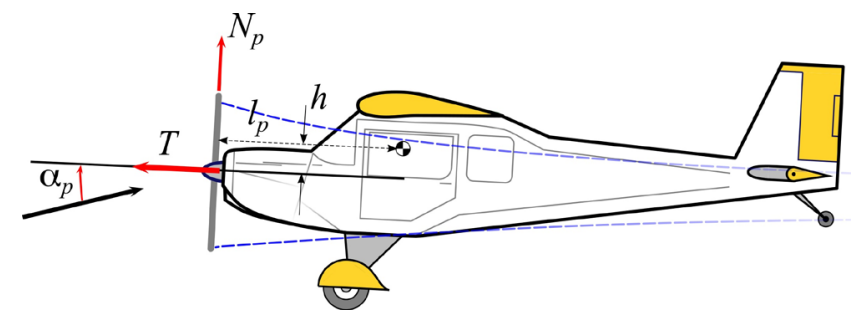

Fig. 3. Typical effects of a propeller on aircraft longitudinal stability.

Direct effects can be easily calculated once the propeller characteristics are known, since their formulation is

$$
M_{p}=T \times h+N_{p} \times l_{p}
$$

while indirect effects are quite hard to predict [11].

In the IRON innovative aircraft configuration, the motors and propellers are mounted on the horizontal tailplane wingtips. The following effects are anticipated:

- asymmetric thrust distribution due to wing and canard wakes impacting on propellers' disks;

- horizontal tailplane load varying with propeller load due to the flow swirl inducing vertical velocities at tailplane;

- different propellers thrust and normal force, even with identical propulsive units, due to the above-mentioned aerodynamic effects.

These are illustrated in Fig. 4. 


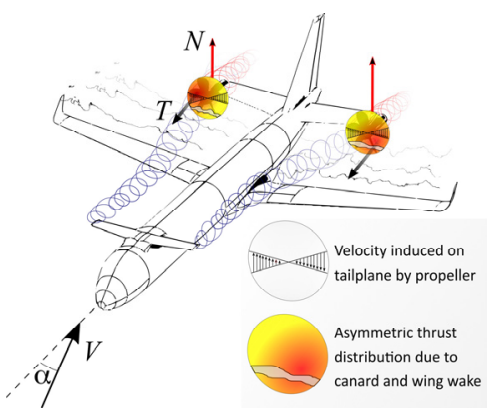

Fig. 4. Illustration of aero-propulsive interactions on the IRON innovative aircraft.

\subsection{Engine deck scaling workflow}

From the original engine deck provided by GE Avio, two flight conditions have been selected to be reproduced in the wind tunnel: a typical climb (MXCL) and a typical cruise (MXCR) condition. From these conditions, model's scale and wind tunnel settings, the scaled engine torque, power and RPM are derived. These are used to choose a motor as a component off-the-shelf.

As scaled engine an electric motor has been chosen, because of the high scalability, availability, versatility and safety of such a machine. Each motor has its own characteristics as operating voltage and current per torque load. These determine to size the power, acquisition and control system.

Similarly, with the assigned flight conditions, propeller coefficients and diameter, the scaled propeller can be designed. This workflow is resumed in the infographic of Fig. 5.
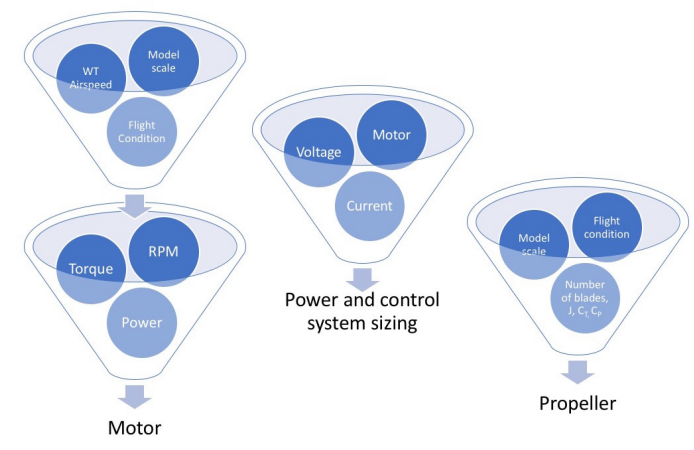

Fig. 5. Engine deck scaling workflow.

\section{Electric motor characteristics}

The selected electric motor is the Lehner 2280/40 LK, shown in Fig. 6. The manufacturer provides performance tables at constant voltage. Each table lists absorbed current, input power, RPM, torque, output (shaft) power, and efficiency. It resulted that this motor has a constant $K_{v}<200 \mathrm{Volt} / \mathrm{RPM}$, ideal for relatively high torques and low speeds. Several performance tables have been collected and processed with MATLAB $^{\circledR}$ to generate motor maps. The classic torque vs RPM map with motor's efficiency contour is shown in Fig. 7. Any propeller operating point falling within this map is reproducible in the wind tunnel, since these combinations of torque load and RPM are within the motor's limits. 

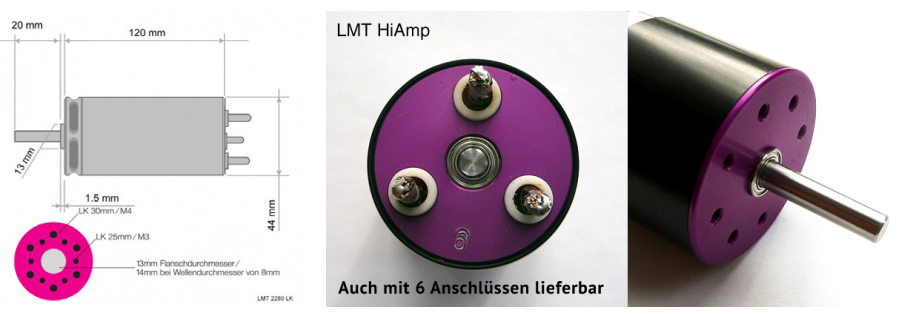

Fig. 6. Lehner 2280/40 LK (air cooled) electric motor.

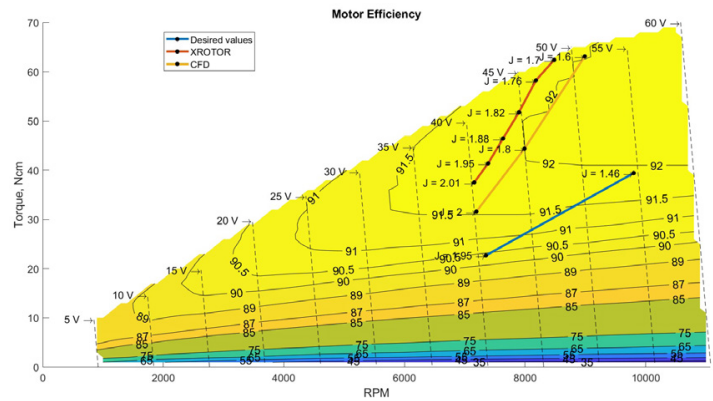

Fig. 7. Scaled motor map.

Similarly, the motor map of Fig. 8 shows the electric current as function of RPM (abscissa), applied voltage (dashed lines) and shaft power (iso-lines representing the motor load). This chart has been useful to size the power system. Again, any propeller operating point falling into this chart can be replicated in the wind tunnel. The markers indicated as "desired values", "XROTOR" and "CFD" are the propeller operating points predicted by the engine deck, XROTOR and RANS analyses respectively for the MXCL flight condition. If the CFD validation, described in the next section, is accurate, it will not be possible to operate within the whole desired advance ratio range $(1.5 \leq J \leq 2.0)$.

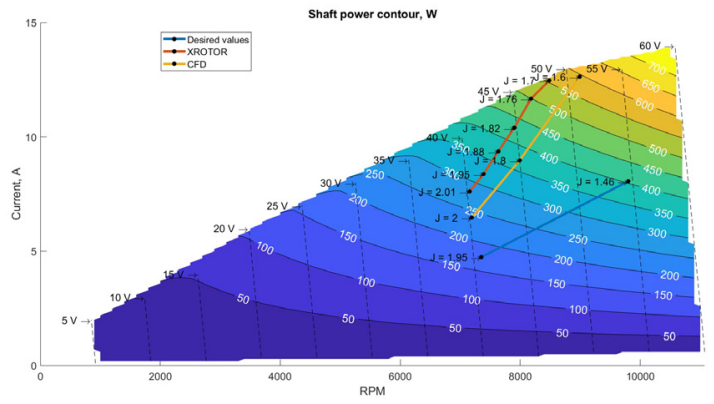

Fig. 8. Alternative scaled motor map used to size the power system.

\section{Propeller design}

For the selected flight conditions, no available RC propeller seems to be fit for the expected torque loads and RPM. Therefore, the propeller must be designed. It is quite challenging to achieve both high thrust coefficient and efficiency, because of the low Reynolds number (less than 100000) expected on the scaled propeller with $14.6 \mathrm{~cm}$ diameter.

XROTOR has been chosen as design software, since it is believed to be the best propeller design package available to the public, especially if accurate section data are loaded [12]. The design workflow is shown in Fig. 9 and described in the following. 


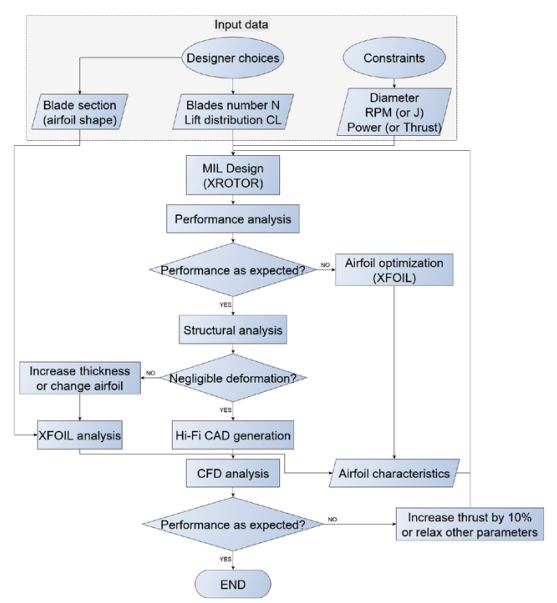

Fig. 9. Propeller design workflow.

From design requirements (diameter, RPM, power or thrust), the blade is designed with XROTOR aiming for minimum induced loss for a given operating point. Then, the propeller is analysed on a range of advance ratios. If the performance is inadequate, an optimisation loop on the blade section may be performed with XFOIL and MATLAB ${ }^{\circledR}$. At the end of the aerodynamic design, a simple structural analysis checks if the blade is strong enough for the expected aerodynamic and centrifugal loads, otherwise it is suggested to increase the blade thickness and the workflows go back to the design routine. At the end of the design process, a high-fidelity CAD is semi-automatically generated. This 3D geometry is ready for advanced numerical analyses and manufacturing.

As concern the blade section, several typical propeller airfoils have been analysed. The MATLAB $^{\circledR}$ routine has been run to perform a single objective optimisation, aiming to minimize drag at the desired blade lift coefficient. The airfoil is parameterised with the PARSEC technique [13] and it is optimised with a genetic or a particle swarm algorithm, where XFOIL is called on parallel workers to perform viscous analyses at the selected Reynolds number and lift coefficient.

The optimised propeller must be feasible to manufacture and to operate. The typical optimisation output provided a rear shape that may result too fragile for the application (Fig. 10). Therefore, the SDA 1075 airfoil [10] has been chosen, with an increased thickness $(\approx 30 \%)$ at blade root and a thicker trailing edge at tip.

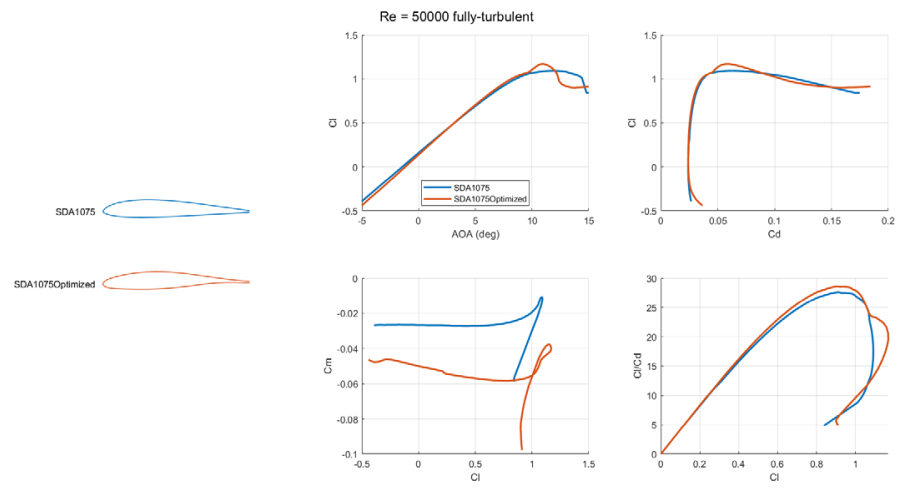

Fig. 10. Blade section optimisation. 
RANS analyses are performed with STAR-CCM+ at the end of the design process. The propeller rotation is simulated with the moving reference frame technique [14]. The flow field is stationary, fully turbulent (k- $\omega$ model), discretized with more than 7 million polyhedral cells for a six-bladed rotor (Fig. 11).
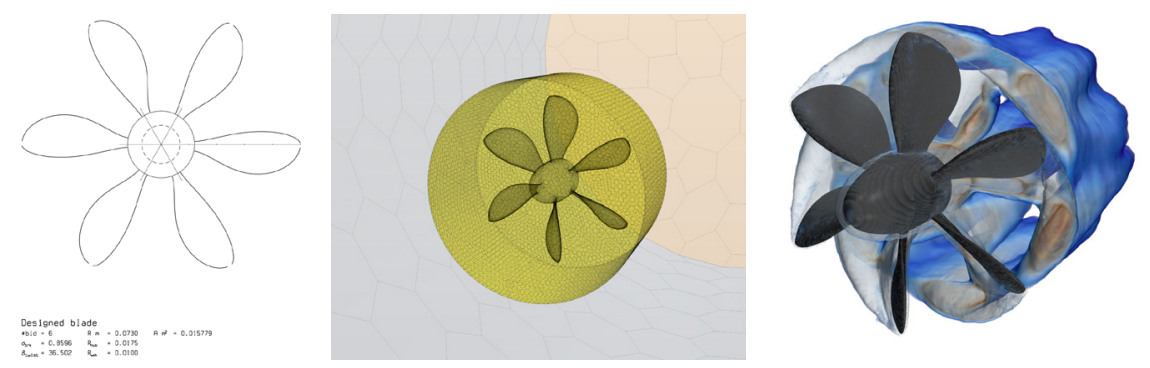

Fig. 11. Propeller CFD RANS validation.

As XROTOR underpredicts thrust by $10 \%$ [12], after several design iterations it was chosen to aim at $110 \%$ of the desired thrust to get satisfactory performance. Figure 12a-b shows the thrust and power coefficients of the propeller designed for the MXCL condition with data predicted by XROTOR (blue line), CFD (red line with markers) and the target values (the green, shortest line). The $C_{T}$ values obtained with the CFD are fairly close to the target values, whereas the $C_{P}$ values are always higher. As expected, the max efficiency calculated with both tools is lower than desired, about 0.2 for the CFD data (Fig. 12c). An analysis of the blade aerodynamic load (Fig. 12d) shows that the lift coefficient distribution is as desired, i.e. constant for a large range of the blade span, although the achieved values are less than those predicted by XROTOR.

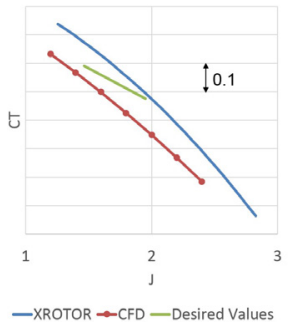

(a)

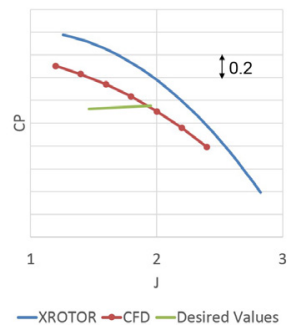

(b)

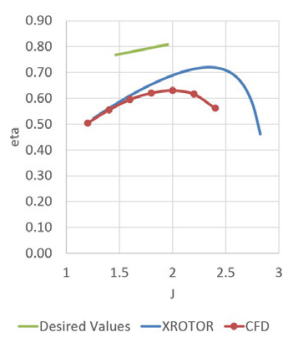

(c)

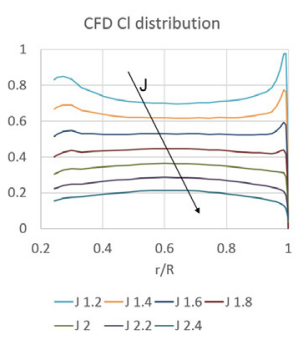

(d)

Fig. 12. Propeller analysis: (a) thrust coefficient; (b) power coefficient; (c) efficiency; (d) spanwise lift coefficient distribution evaluated with CFD.

\section{Wind tunnel hardware upgrades}

The power supply, data acquisition and motor control scheme is shown in Fig. 13. The propulsive system will be instrumented with load cells to measure propeller thrust and normal forces, a temperature sensor to monitor the motor's temperature during operations, a RPM counter that will be probably installed on one of the motor cooling windows, and an electronic speed controller (ESC) MGM Compro HBC series (air cooled). Data acquisition and motor control will be performed by a National Instruments ${ }^{\mathrm{TM}}$ USB-6341 card. Power supply will be provided by four $12 \mathrm{~V}, 70 \mathrm{Ah}$ lead batteries that will generate constant voltage for a reasonable amount of time and eventually absorb power in the case of propeller braking. 


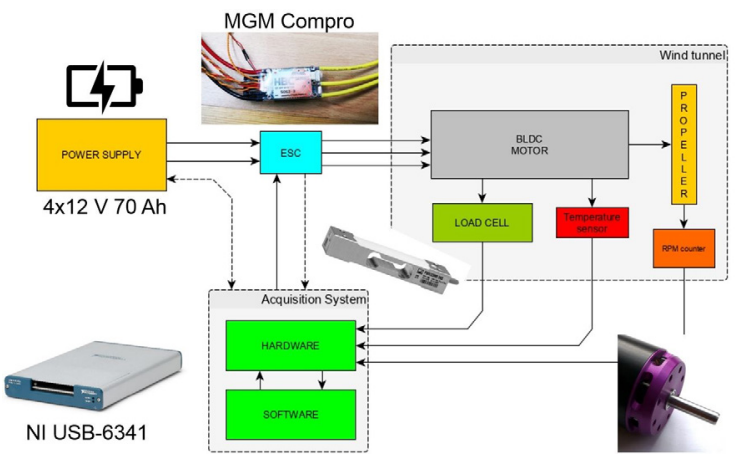

(a)

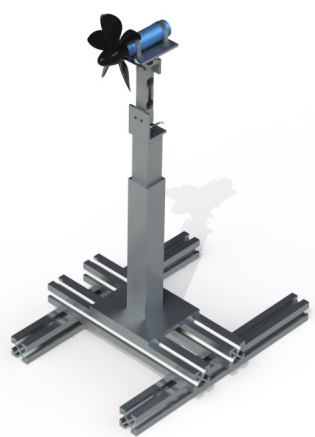

(b)

Fig. 13. (a) Data acquisition and control scheme; (b) motor support stand.

The motor support system, manually operated with wind tunnel turned off, is designed to move the motors in the correct position in close proximity to the aircraft, without contact, to directly measure propeller thrust and normal force. It provides clamps for the motors as well as housing for the ESC and the load cells. The motor can be moved along three axes and rotated about the local pitch axis, following the different attitudes of the aircraft.

The IRON project has received funding from the Clean Sky 2 Joint Undertaking under the European Union's Horizon 2020 research and innovation program under Grant Agreement $n^{\circ}$ 699715. The authors are grateful to the partners of the IRON consortium for their contributions and feedback.

\section{References}

[1] S. Corcione, V. Trifari, F. Nicolosi, V. Cusati, D. Ciliberti, P. Della Vecchia, Proc. 2019 EASN Int. Conf. (to be published)

[2] F. Nicolosi, S. Corcione, V. Trifari, V. Cusati, M. Ruocco, P. Della Vecchia, 2018 Aviation Tech., Integr., and Ops. Conf. (2018)

[3] F. Nicolosi, S. Corcione, P. Della Vecchia, V. Trifari, M. Ruocco, 31st ICAS Congr. (2018)

[4] F. Nicolosi, S. Corcione, V. Trifari, P. Della Vecchia, A. De Marco, AIAA Scitech 2019 Forum (2019)

[5] S. Corcione, F. Nicolosi, P. Della Vecchia, D. Ciliberti, V. Cusati, AIAA Aviation 2019 Forum (2019)

[6] V. Cusati, F. Nicolosi, S. Corcione, D. Ciliberti, P. Della Vecchia, AIAA Aviation 2019 Forum (2019)

[7] J.B. Barlow, W.H. Rae, A. Pope, Low-speed wind tunnel testing (Wiley, New York, 1999)

[8] R.M. Bass, 24th AIAA Aerosp. Sci. Meet. (1986)

[9] J.B. Brandt, M.S. Selig, 49th AIAA Aerosp. Sci. Meet. (2011)

[10] R.W. Deters, G.K. Ananda, M.S. Selig, 32nd AIAA Appl. Aerodyn. Conf. (2014)

[11] C.D. Perkins, R.E. Hage, Airplane Performance Stability and Control (Wiley, New York, 1949)

[12] P.C. Klein, Parametric Modeling and Optimization of Advanced Propellers for NextGeneration Aircraft (TU Delft, 2017)

[13] H. Sobieczky, Notes on Numerical Fluid Mechanics, 71-88 (1998)

[14] STAR-CCM+ 13.04 manual (2018) 